\title{
Efficacy of Common Reagents for Killing Ticks in the Ear Canal
}

\author{
Nicklas C. Orobello, MD; Carolyn O. Dirain, PhD (D; Phillip E. Kaufman, PhD; Patrick J. Antonelli, MD $\mathbb{D}$
}

Objective: To determine if widely available solutions can effectively kill ticks that may be found in the human ear canal.

Methods: This study was prospective, controlled and blinded animal study. Lone star ticks (Amblyomma americanum), both nymphal and adult, were submerged in one of four preparations (acetone, isopropyl alcohol 70\%, ethanol 95\%, or 4\% lidocaine) in test tubes ( $\mathrm{n}=20$ per group) for 20 minutes. Ticks were agitated by intermittent probing. Activity of the nymphal ticks was directly observed while those of the adult ticks was video-recorded during the exposure period. Two blinded investigators viewed the videos of adult ticks and during the exposure period to determine the time until death (ie, movement cessation). Mortality was assessed immediately after exposure, and confirmed 24 and 48 hours after exposure.

Results: Acetone killed ticks most rapidly (nymph mean time $=185.1 \mathrm{~s}$; adult mean time $=562.9 \mathrm{~s}$ ). Isopropyl alcohol $70 \%$ (nymphs, $328.9 \mathrm{~s}$; adults, $1128.4 \mathrm{~s}$ ) and ethanol 95\% (nymphs, $294 \mathrm{~s}$; adults, $1129.4 \mathrm{~s}$ ) took longer to kill the ticks. All ticks treated with $4 \%$ lidocaine survived. These differences were significant (nymphs, $P<.0001 ;$ adults, $P<.0001$ ).

Conclusions: Acetone was the fastest acting and most effective reagent, followed by ethanol and isopropyl alcohol. These solutions may prove useful in otoacariasis with an intact tympanic membrane.

Key Words: Tick, foreign body, ear canal, treatment, acetone.

Level of Evidence: N/A

\section{INTRODUCTION}

The human external auditory canal is vulnerable to arachnid incursion. ${ }^{1,2}$ These cases often present with otalgia and tinnitus, ${ }^{2-5}$ which can cause significant emotional distress for the patient. ${ }^{6}$ Prompt killing of the tick prior to removal may help to alleviate this distress, reduce the chance of transmission of infectious diseases and of secondary complications, and facilitate the removal process. As compression of the tick body can promote transmission of microbes into the host, ${ }^{7,8}$ and the tick's head can be difficult to grasp (eg, in the anterior sulcus), optimal killing of the tick would involve the tick detaching from the host, facilitating extraction. Furthermore, an ideal preparation would have minimal host toxicity, as the bite effectively creates an open wound in the canal or a perforation of the tympanic membrane.

We have previously assessed the efficacy of common reagents to kill a variety of insects and ticks. ${ }^{1}$ In contrast to insects, ticks were not readily killed with common reagents. However, the exposure was limited to 3 minutes.

This is an open access article under the terms of the Creative Commons Attribution-NonCommercial-NoDerivs License, which permits use and distribution in any medium, provided the original work is properly cited, the use is non-commercial and no modifications or adaptations are made.

From the Department of Otolaryngology-Head and Neck Surgery (N.C.O, C.O.D., P.J.A.); and the Entomology and Nematology (P.E.K.), University of Florida, Gainesville, FL

Editor's Note: This Manuscript was accepted for publication 00 Month 2018

Financial support for this study was provided by the University of Florida. None of the authors have any relevant financial conflicts to disclose.

This study was presented at the AAO-HNSF Annual Meeting and Oto Expo, September 21-24, 2014, Orlando, Florida.

Send correspondence to Patrick J. Antonelli, MD, Box 100264, 1345 Center Drive Gainesville, FL 32610. Email: pa@ufl.edu

DOI: 10.1002/lio2.217
Based on additional personal experience with otoacariasis, we sought to evaluate the effectiveness of commonly available preparations for killing ticks over a longer exposure period.

\section{MATERIALS AND METHODS}

\section{Study Design}

The lone star tick (Amblyomma americanum) was chosen for this study because they have been most implicated in cases of parasitism on humans. ${ }^{9-11}$ Both nymphal and adult ticks were used in this study because lone star ticks bite humans in all three life stages, and both could present as foreign bodies to the ear canal. ${ }^{12,13}$ Ticks were purchased from the Oklahoma State University Department of Entomology (Stillwater, OK). All ticks were housed in optimal conditions, as instructed by an entomologist, from 12 hours prior to experimentation to completion of the study.

Both nymphal and adult ticks were randomly allocated to four exposure groups. These four exposure reagents were chosen based on the most efficacious reagents from our previous study (ie, 95\% ethanol and 70\% isopropyl alcohol), additional trial and error with clinical cases (ie, acetone), and input from an entomologist (4\% lidocaine). ${ }^{1}$ Allocation of the ticks to each of the four experimental groups was concealed, so that the experimenter did not know in which preparation the next tick would be treated. Twenty nymphs and 20 adults were used in each exposure group ( 80 nymphs and 80 adults in total). Results of 3 adult ticks in each exposure group were discarded due to technical difficulties with the video camera, leaving 17 adult ticks in each of the exposure groups.

\section{Assessment of Acaricidal Activity of the Solutions}

Test solutions (acetone, 70\% isopropyl alcohol, and 4\% lidocaine) were purchased from commercial vendors with the 
exception of ethanol. Pure ethanol was diluted to $95 \%$ ethanol using distilled water.

Ticks were individually submerged in $1.5 \mathrm{~mL}$ of the respective preparation in a 5 -mL test tube for 20 minutes. Ticks were agitated with standardized probing intermittently throughout the exposure period. Activity of the adult ticks was video-recorded during the exposure period. After treatment, two blinded investigators viewed the videos and recorded the ticks' exposure time until death, as determined by movement cessation.

Nymphal ticks were directly observed, as they are too small to visualize on the video camera. A blinded investigator exhaled on all ticks immediately after exposure, 24 hours after, and 48 hours after exposure to confirm mortality. Expired air provides warm, humid air and carbon dioxide-a novel chemoattractant for lone star ticks-both of which stimulate tick activity. ${ }^{14,15}$ Upon exhalation, increased tick activity was observed in ticks that survived treatment. Conversely, ticks that did not respond to expired air were deemed dead.

\section{Statistical Analysis}

Kaplan-Meier survival curves were fitted by solution type for adult and nymphal ticks. If a tick was still alive after a 20-minute exposure to a preparation, the tick had a censored survival time. Results were charted with the horizontal axis representing the survival time ( $t$ ) in seconds, and the vertical axis represented the probability of surviving for a time greater than or equal to $t$ seconds. The median survival time was reported as the time at which the probability of surviving to or past time $t$ was 0.5 . The log-rank test was used to test the null hypothesis that there were no differences in survival between the four solutions. If this overall test result was significant, all the pairwise comparisons were conducted. These pairwise comparisons were considered statistically significant only if the $P$ value was less than .0125 because the Bonferroni correction was used for multiple testing. The Bonferroni correction was performed to protect the overall type I error rate (significance level) at 0.05 .

\section{RESULTS}

Three of the four test preparations exhibited effective acaricidal activity (Figs. 1 and 2). Pure acetone killed all adult and nymphal ticks, while $95 \%$ ethanol and $70 \%$ isopropyl alcohol each killed all but two adult ticks and all nymphal ticks in the allotted time. The two adult ticks surviving exposure to 95\% ethanol and 70\% isopropyl alcohol were confirmed dead 24 hours after exposure. Conversely, 4\% lidocaine exhibited no acaricidal activity. Acetone proved to be the most effective acaricidal agent, killing both adult ticks (mean time, $562.9 \mathrm{~s}$ ) and nymphal ticks (mean time, 185.1 s) significantly quicker than did the other tested preparations (nymphs, $P<.0001$; adults, $P<.0001$ ).

Bonferroni's multiple comparisons test showed the differences in mean survival time, acetone versus $70 \%$ isopropyl alcohol and acetone versus $95 \%$ ethanol, to be significant in both adult and nymphal ticks $(P<.0001)$. However, the difference in mean survival time between $70 \%$ isopropyl alcohol and 95\% ethanol was not significant for either adult or nymphal ticks.

\section{DISCUSSION}

Ticks may present as foreign bodies of the external ear canal, causing unpleasant symptoms such as otalgia and tinnitus. ${ }^{2-5}$ Worse yet, otoacariasis may lead to secondary infections such as Lyme Disease and Rocky Mountain spotted fever, as well as severe complications including sensorineural hearing loss and vertigo. ${ }^{9,16,17}$

Effective clinical management of otoacariasis is important in improving patients' quality of life and preventing serious secondary complications. We were unable to identify an effective acaricidal agent in our previous study. ${ }^{1}$ We hypothesized that increasing the exposure period of the ticks to the reagents would improve their acaricidal activity. Ethanol and isopropyl alcohol effectively killed nearly all of the ticks, supporting our initial hypothesis that these agents are acaricidal given a prolonged exposure time. Acetone was the most effective reagent, quickly killing all tested ticks.

Lidocaine did not exhibit any acaricidal or paralyzing activity. Contrary to Somayaji and Rajeshqari's previous report, ${ }^{2}$ both nymphal and adult ticks remained highly active throughout the lidocaine exposure period. Hence, we concluded that lidocaine is not an effective agent, as it would neither facilitate extraction nor kill the ticks.

Clinically, acetone may be the reagent of choice in cases of otoacariasis because acetone seems to be

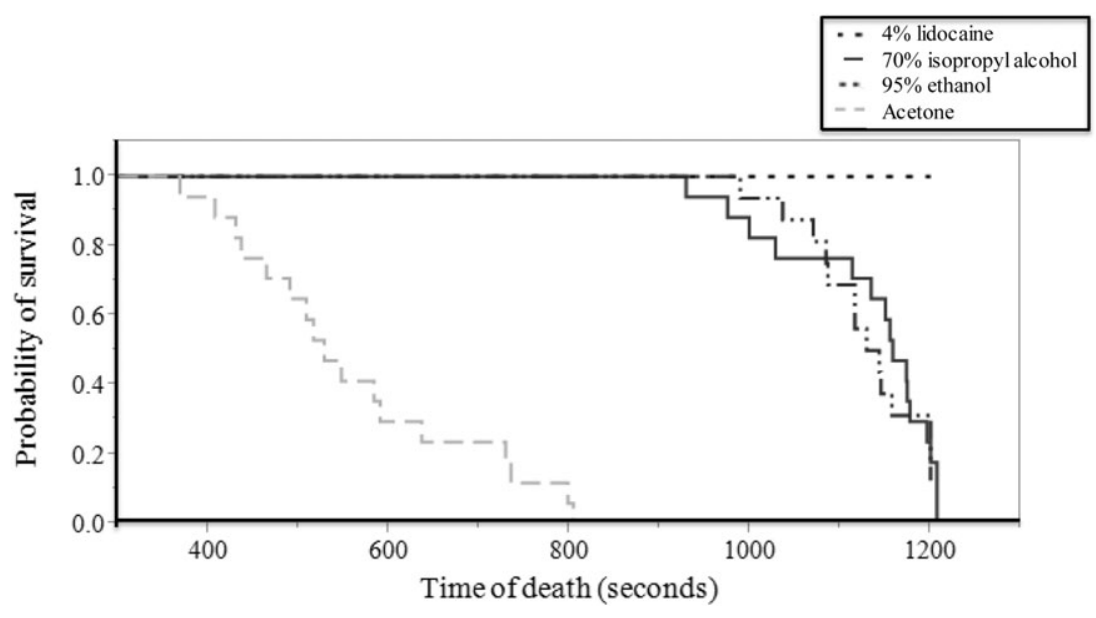

Fig. 1. Survival of adult lone star ticks (Amblyomma americanum). Bonferroni's multiple comparisons test $(P<.0001)$. 


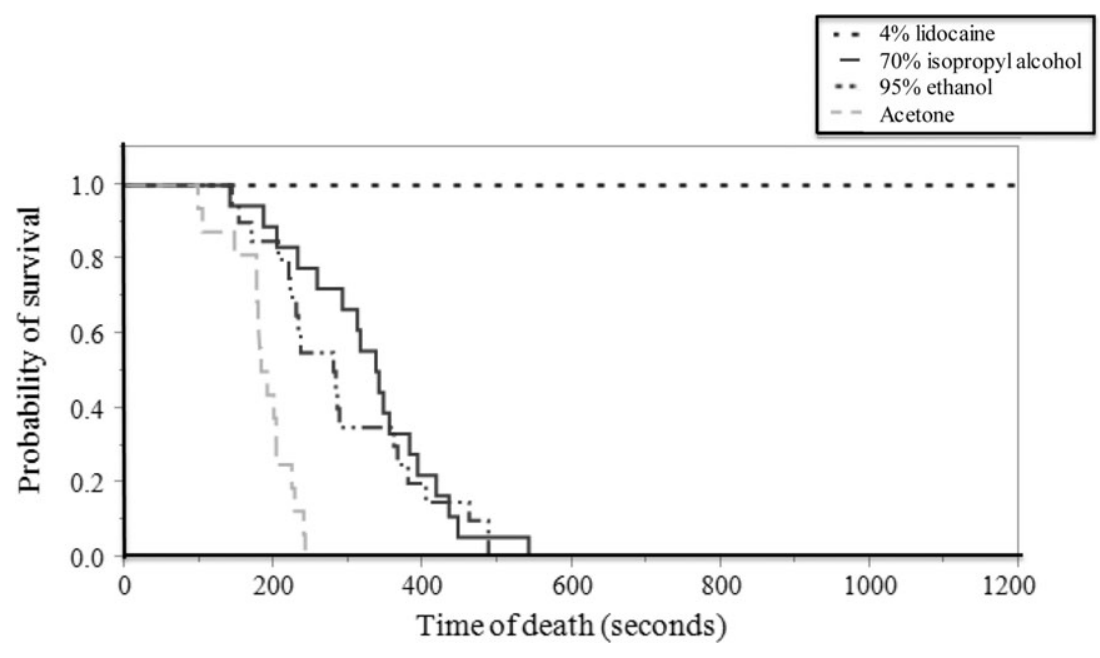

Fig. 2. Survival of nymphal lone star ticks (Amblyomma americanum). Bonferroni's multiple comparisons test $(P<.0001)$.

uniformly acaricidal in the present study. Acetone works significantly quicker than ethanol and isopropyl alcohol. Thus, the external ear canal can be treated with acetone for a shorter period of time. Moreover, acetone is minimally toxic and is not considered mutagenic or carcinogenic when applied to the skin. Chronic skin exposure to acetone can cause irritant dermatitis, but short-term dermal exposure to acetone is well-tolerated. ${ }^{14}$ Acetone has been used previously in the ear canal of patients to facilitate the removal of superglue (cyanoacrylate), ${ }^{15,16}$ styrofoam (polysterene), ${ }^{17}$ and chewing gum. ${ }^{18}$ In these reports, a few drops of pure acetone was instilled directly into the ear canal or a cotton ball was soaked in pure acetone and the cotton ball was left in the ear canal. The duration of acetone in the ear canal in these studies was anywhere from 30 seconds to 10 minutes. These studies concluded that acetone was relatively safe, with no considerable damage to the ear canal skin or tympanic membrane. In the present study, acetone killed all nymphal and adult ticks in about three and nine minutes, respectively. This amount of time is within the 10 minutes that Abadir et al. ${ }^{15}$ used acetone in the external meatus in one of their two cases. However, although the toxic effects of acetone on the external ear may be local and minimal, ${ }^{15-17}$ research regarding the potential ototoxicity (ie, toxicity to the inner ear) of acetone is lacking. Due to potential perforation of the tympanic membrane by the tick bite, acetone should be used with caution in the presence of a non-intact tympanic membrane. Although acetone evaporates very rapidly, irrigation of the ear canal with sterile water ${ }^{17}$ may help minimize the potential adverse effects of acetone.

Ethanol and isopropyl alcohol also effectively killed nearly all of the ticks, but it took twice as long compared to that of acetone. Furthermore, ethanol has been shown to cause erythema and mild edema in the mucosa of the middle ear cavities of sand rats treated with $70 \%$ ethanol as antiseptic, ${ }^{19}$ while $90 \%$ to $95 \%$ isopropyl alcohol used as a prophylaxis for otitis externa dries the ear canal, and can cause stinging and local irritation. ${ }^{20}$ Both ethanol and isopropyl alcohol have the potential for ototoxicity. ${ }^{21-23}$ Again, due to potential perforation of the tympanic membrane by the tick bite, the ototoxicity of these reagents must be weighed against their benefits.

Accordingly, all of these acaricidal solutions should be used with caution in the presence of a non-intact tympanic membrane due to potential for ototoxicity.

\section{CONCLUSIONS}

Acetone was the fastest acting and most effective reagent, followed by ethanol and isopropyl alcohol. These solutions may prove useful in otoacariasis with an intact tympanic membrane.

\section{ACKNOWLEDGMENTS}

We thank Maria Raye Anne Ng and Jerin Joseph for reviewing and scoring the tick videos.

\section{BIBLIOGRAPHY}

1. Antonelli PJ, Ahmadi A, Prevatt A. Insecticidal activity of common reagents for insect foreign bodies of the ear. Laryngoscope 2001;111(1):15-20.

2. Somayaji KS, Rajeshwari A. Human otoacariasis. Indian $J$ Otolaryngol Head Neck Surgery 2007;59(3):237-239.

3. Erkalp K, Kalekoglu Erkalp N, Ozdemir H. Acute otalgia during sleep (live insect in the ear): a case report. Agri 2009;21(1):36-38.

4. Supiyaphun P, Sukumanpaiboon P. Acute otalgia: a case report of mature termite in the middle ear. Auris Nasus Larynx 2000;27(1):77-78.

5. Arnold A, Arnold W. Images in clinical medicine. A tickling in the ear. $N$ Engl J Med 2012;367(12):e17.

6. Ng WY, Chua BE, Hardy TA, Wechsler D, Reddel SW. Bilateral homonymous superior quadrantanopia after traumatic attempts to remove a cockroach impacted in the external auditory canal. Med J Aust 2011;194(8): $420-422$.

7. Miyamoto K, Hashimoto Y. [Prevention of Lyme borreliosis infection after tick bites]. Kansenshogaku Zasshi 1998;72(5):512-516.

8. Piesman J, Dolan MC. Protection against lyme disease spirochete transmission provided by prompt removal of nymphal Ixodes scapularis (Acari: Ixodidae). J Med Entomol 2002;39(3):509-512.

9. Armstrong PM, Brunet LR, Spielman A, Telford SR, 3rd. Risk of Lyme disease: perceptions of residents of a Lone Star tick-infested community. Bull World Health Organ 2001;79(10):916-925.

10. Felz MW, Durden LA, Oliver JH, Jr. Ticks parasitizing humans in Georgia and South Carolina. J Parasitol 1996;82(3):505-508. 
11. Goddard J. A ten-year study of tick biting in Mississippi: implications for human disease transmission. $J$ Agromedicine 2002;8(2):25-32.

12. Childs JE, Paddock CD. The ascendancy of Amblyomma americanum as a vector of pathogens affecting humans in the United States. Ann Rev Entomol 2003;48:307-337.

13. Goddard J, Varela-Stokes AS. Role of the Lone Star tick, Amblyomma americanum (L.), in human and animal diseases. Vet Parasitol 2009, 160(1-2):1-12.

14. Spyker DaS, JB Oxygenated compounds: Alcohols, glycols, ketones, and esters. In: Sullivan JB KG, ed. Hazardous Materials Toxicology-Clinical Principles of Environmental Health. Baltimore, MD: Williams \& Wilkins; 1992:1105-1116.

15. Abadir WF, Nakhla V, Chong P. Removal of superglue from the external ear using acetone: case report and literature review. $J$ Laryngol Otol. 1995;109(12):1219-1221.

16. Gogia S, Agarwal A. Removal of superglue from the external ear using acetone: a case report. Egyptian J Otolaryngol 2016;32(2):116-117.
17. White SJ, Broner S. The use of acetone to dissolve a Styrofoam impaction of the ear. Ann Emerg Med 1994;23(3):580-582.

18. Chisholm EJ, Barber-Craig H, Farrell R. Chewing gum removal from the ear using acetone. J Laryngol Otol 2003;117(4):325.

19. Perez R, Freeman S, Sohmer H, Sichel J-Y. Vestibular and Cochlear Ototoxicity of Topical Antiseptics Assessed by Evoked Potentials. Laryngoscope 2000;110(9):1522-1527.

20. Osguthorpe JD, Nielsen DR. Otitis externa: review and clinical update. Am Fam Physician 2006;74(9):1510-1516.

21. Morizono T, Sikora MA. Ototoxicity of ethanol in the tympanic cleft in animals. Acta Otolaryngol 1981;92(1-2):33-40.

22. Singh S, Blakley B. Systematic review of ototoxic pre-surgical antiseptic preparations - what is the evidence? J Otolaryngol Head Neck Surgery 2018;47(1):18.

23. Spandow O, Anniko M, Moller AR. The round window as access route for agents injurious to the inner ear. Am J Otolaryngol 1988;9(6):327-335. 